\title{
Efficient Signal Detection for Space-Time Block Coding Over Time-Selective Fading Channels
}

\author{
Tao Cui \\ Department of Electrical Engineering \\ California Institute of Technology \\ Pasadena, CA, USA 91125 \\ Email: taocui@caltech.edu
}

\author{
Chintha Tellambura \\ Department of Electrical and Computer Engineering \\ University of Alberta \\ Edmonton, AB, Canada T6G 2V4 \\ Email: chintha@ece.ualberta.ca
}

\begin{abstract}
In this paper, we consider the signal detection for space-time block coding over time-selective fading channels. We derive a general maximum likelihood (ML) decision rule for space-time block coding (STBC). The resulting detector is valid for any number of receive antennas and for all STBC systems that have the linear dispersion property. The detector results in a quadratic minimization problem, which can be solved efficiently by sphere decoding or nulling-and-cancelling detection. For orthogonal STBC systems, we also propose a suboptimal detector using the principle of parallel interference cancellation (PIC) that is valid for systems with an arbitrary number of receive antennas.
\end{abstract}

\section{INTRODUCTION}

Space-time block coding (STBC) [1]-[3] is used in multiantenna wireless systems to effectively utilize diversity gains. Orthogonal STBC (OSTBC), a subset of STBC systems, achieves full transmit diversity and is amenable to simple linear maximum-likelihood (ML) decoding if the channel state information (CSI) is known at the receiver. Non-orthogonal STBC (NOSTBC) has also been proposed to achieve full rate transmission, which can be decoded using a pairwise ML decoder. The linear ML decoder for OSTBC and the pairwise ML decoder for NOSTBC require that the channel remains static over the length of the STBC codeword. However, for certain vehicle speeds, time selective or fast fading channels arise. The time-selectivity of the channel will destroy the orthogonality of the OSTBC channel matrix at the receiver; consequently, the linear ML decoder results in an irreducible error floor [4], [5]. Likewise, for the NOSTBC case, the pairwise ML decoder is no longer valid [6]. These facts have motivated the development of signal detection algorithms for STBC over time-selective fading channels.

Several such detectors have been given in [4]-[6] for both OSTBC and NOSTBC over time-selective fading channels. In [4], [5], detectors for $\mathcal{H}_{i}$ and $\mathcal{G}_{i}$ codes are derived, and they use the principle of parallel interference cancellation (PIC). A least-squares (LS) detector for non-orthogonal STBC is given in [6]. However, these detectors are not derived in the ML sense, and each detector is only designed for a specific case of space-time code. Moreover, all of the detectors [4]-[6] are designed for the case of one receive antenna. No general ML detection rule has been derived in [4]-[6] for an arbitrary STBC system and an arbitrary number of receive antennas.
Furthermore, the use of PIC and LS results in suboptimal detectors that may exhibit an irreducible error floor in high SNR.

In this paper, we systematically derive a general ML decision rule for an arbitrary STBC system that has the linear dispersion (LD) property [7] over a time-selective fading channel. Note that in the LD codes, the transmitted codeword is a linear combination of certain basis matrices. Both OSTBC and NOSTBC systems can be viewed as subsets of LD codes. Our ML decision rule results in a quadratic discrete optimization problem. The computational complexity of exhaustive search for the optimal solution grows exponentially with the dimension of the OSTBC system. What is therefore needed is an efficient search method; the sphere decoding (SD) [8] paradigm provides such a method. Note that SD provides the same optimal solution as the exhaustive search method, but results in much reduced complexity. Some recent results on SD can be found in [9]-[11]. On the other hand, the discrete optimization problem can be suboptimally solved by the vertical-bell labs layered space-time (V-BLAST) detection algorithm [12], which is a nulling-and-cancelling detector. In addition to the two SD and V-BLAST based detectors for STBC over time-selective channels, we also develop a suboptimal PIC decoder for OSTBC systems by exploiting the fact that the channel matrix is almost orthogonal. Unlike the PIC detectors in [4]-[6], our PIC detector does not depend on specific space-time codes and is applicable for any number of receive antennas.

The rest of the paper is organized as follows. The timeselective channel model and STBC systems are introduced in Sections II and III. In Section IV, the general ML detector is derived and SD is used to solve the detector. A suboptimal PIC detector is also derived for OSTBC. Numerical results are illustrated in Section V, and conclusions are drawn in Section VI.

Notation: $E\{\cdot\},(\cdot)^{*},(\cdot)^{T},(\cdot)^{H}$ and $(\cdot)^{\dagger}$ denote expectation, complex conjugation, transpose, conjugate transpose and Moore-Penrose pseudo-inverse, respectively. The imaginary unit is $\jmath=\sqrt{-1}$. The $N \times N$ identity matrix is $\mathbf{I}_{N}$. The Kronecker delta is $\delta_{i, j}=1$ if $i=j$ and $\delta_{i, j}=0$ if $i \neq j$ where $i, j \in \mathbb{Z}$. 


\section{Time-Selective MiMO Channel Model}

We consider a MIMO system with $N_{t}$ transmit and $N_{r}$ receive antennas. Each transmitted codeword (matrix) has $T$ time slots, each of which has a duration of $T_{s}$, the symbol interval. We consider a frequency-flat Rayleigh fading MIMO channel resulting from a rich scattering environment. Throughout this paper, we assume that the perfect CSI is available at the receiver. For instance, the time-varying channel can be estimated using the algorithm in [13]. The received signal at the $j$ th receive antenna at time slot $t$ in the $n$th block can be written as

$$
r_{t, j}=\sum_{i=1}^{N_{t}} h_{i, j}[t] s_{t, i}+w_{t, j}
$$

where $h_{i, j}[t]$ denotes the path gain from the $i$ th transmit antenna to the $j$ th receive antenna, $s_{t, i}$ is transmitted by the $i$ th antenna in the $t$-th time slot, and $w_{t, j}$ is the complex additive white Gaussian noise at the $j$ th receive antenna with zero mean and variance $\sigma_{n}^{2}$. The codeword index $n$ is dropped for notational brevity. The fading channel is time-selective. All the path gains are statistically independent and have the identical time correlation function $R_{h}(\tau)$. Typically, when Jakes' model is used, the autocorrelation is given by

$$
\begin{aligned}
R_{h}[m] & =E\left\{h_{i, j}[i] h_{i^{\prime}, j^{\prime}}^{*}[i+m]\right\} \\
& =\delta_{i, i^{\prime}} \delta_{j, j^{\prime}} \sigma_{h}^{2} J_{0}\left(2 \pi m f_{d} T_{s}\right)
\end{aligned}
$$

where $\sigma_{h}^{2}$ denotes the power of the path gain, $J_{0}(\cdot)$ is the zeroth-order Bessel function of the first kind, and $f_{d}$ is the Doppler frequency.

\section{LD REPRESENTATION OF STBC}

The transmitted symbols during the $n$th codeword are denoted by the $T \times N_{t}$ matrix $\mathbf{S}=\left[s_{t, i}\right], t=1,2, \ldots, T$ and $i=1,2, \ldots, N_{t}$. For STBC systems, $P$ modulation symbols $\mathbf{x}=\left[x_{1}, x_{2}, \ldots, x_{P}\right]^{T}$ with the same average power $E_{s}=$ $E\left\{\left|x_{p}\right|^{2}\right\}$ are transmitted in each codeword, and $r=P / T$ is the rate of the code. The transmit matrix symbol $\mathbf{S}$ is a function of $\mathbf{x}$. For example, in OSTBC systems, $\mathbf{S}$ has the property

$$
\mathbf{S}^{H} \mathbf{S}=c\left(\sum_{p=1}^{P}\left|x_{p}\right|^{2}\right) \mathbf{I}_{N_{t}} .
$$

where $c=1 / r$. For the Alamouti code [1] or the $\mathcal{G}_{2}$ code in [2], $N_{t}=2, P=2, T=2, c=1$ and $\mathbf{S}$ is given by

$$
\mathbf{S}=\left(\begin{array}{cc}
x_{1} & x_{2} \\
-x_{2}^{*} & x_{1}^{*}
\end{array}\right) \text {. }
$$

For the $\mathcal{G}_{3}$ and $\mathcal{G}_{4}$ codes in [2], $c=2$.

LD STBC systems can be represented as [7]

$$
\mathbf{S}=\sum_{p=1}^{P}\left(\alpha_{p} \mathbf{A}_{p}+\jmath \beta_{p} \mathbf{B}_{p}\right),
$$

where $x_{p}=\alpha_{p}+\jmath \beta_{p}$, and $\mathbf{A}_{q}$ and $\mathbf{B}_{q}$ are called dispersion matrices [7], which characterize a given STBC. For OSTBC, the dispersion matrices satisfy the following:

$$
\begin{aligned}
\mathbf{A}_{i}^{H} \mathbf{A}_{j} & =\mathbf{0}, \mathbf{B}_{i}^{H} \mathbf{B}_{j}=\mathbf{0}, \mathbf{A}_{i}^{H} \mathbf{B}_{j}=\mathbf{0}, i \neq j \\
\mathbf{A}_{i}^{H} \mathbf{A}_{i} & =\mathbf{I}, \mathbf{B}_{i}^{H} \mathbf{B}_{i}=\mathbf{I}, \mathbf{A}_{i}^{H} \mathbf{B}_{i}=\mathbf{B}_{i}^{H} \mathbf{A}_{i} .
\end{aligned}
$$

For the Alamouti code, one of the most well-known OSTBC systems, we have

$$
\begin{aligned}
\mathbf{A}_{1}=\left(\begin{array}{ll}
1 & 0 \\
0 & 1
\end{array}\right), & \mathbf{A}_{2}=\left(\begin{array}{cc}
0 & 1 \\
-1 & 0
\end{array}\right), \\
\mathbf{B}_{1}=\left(\begin{array}{cc}
1 & 0 \\
0 & -1
\end{array}\right), & \mathbf{B}_{2}=\left(\begin{array}{cc}
0 & 1 \\
1 & 0
\end{array}\right) .
\end{aligned}
$$

For the NOSTBC given by [3, Eq. (5)], $N_{t}=4, P=4$, $T=4$, and $\mathbf{S}$ is given by

$$
\mathbf{S}=\left(\begin{array}{cccc}
x_{1} & x_{2} & x_{3} & x_{4} \\
-x_{2}^{*} & x_{1}^{*} & -x_{4}^{*} & x_{3}^{*} \\
-x_{3}^{*} & -x_{4}^{*} & x_{1}^{*} & x_{2}^{*} \\
x_{4} & -x_{3} & -x_{2} & x_{1}
\end{array}\right)
$$

The dispersion matrices $\mathbf{A}_{q}$ can be obtained as

$$
\begin{gathered}
\mathbf{A}_{1}=\left(\begin{array}{cccc}
1 & 0 & 0 & 0 \\
0 & 1 & 0 & 0 \\
0 & 0 & 1 & 0 \\
0 & 0 & 0 & 1
\end{array}\right), \mathbf{A}_{2}=\left(\begin{array}{cccc}
0 & 1 & 0 & 0 \\
-1 & 0 & 0 & 0 \\
0 & 0 & 0 & 1 \\
0 & 0 & -1 & 0
\end{array}\right), \\
\mathbf{A}_{3}=\left(\begin{array}{cccc}
0 & 0 & 1 & 0 \\
0 & 0 & 0 & 1 \\
-1 & 0 & 0 & 0 \\
0 & -1 & 0 & 0
\end{array}\right), \mathbf{A}_{4}=\left(\begin{array}{cccc}
0 & 0 & 0 & 1 \\
0 & 0 & -1 & 0 \\
0 & -1 & 0 & 0 \\
1 & 0 & 0 & 0
\end{array}\right) .
\end{gathered}
$$

The dispersion matrices $\mathbf{B}_{q}$ can be obtained similarly. Clearly, the LD model (5) is general enough to represent existing linear STBC systems.

\section{ML STBC DETECTOR OVER TIME-SELECTIVE FADING \\ CHANNELS}

For each time slot $t,(1)$ can be written in matrix form as

$$
\mathbf{r}[t]=\mathbf{H}^{T}[t] \mathbf{s}[t]+\mathbf{w}[t], \quad t=1, \ldots, T,
$$

where $\mathbf{r}[t]=\left[r_{t, 1}, \ldots, r_{t, N_{r}}\right]^{T}, \mathbf{s}[t]=\left[s_{t, 1}, \ldots, s_{t, N_{t}}\right]^{T}$, $\mathbf{H}[t]=\left[h_{i, j}[t]\right]$ and $\mathbf{w}[t]=\left[w_{t, 1}, \ldots, w_{t, N_{r}}\right]^{T}$. Using (5), we have

$$
\mathbf{s}[t]=\sum_{p=1}^{P}\left(\alpha_{p} \mathbf{a}_{t, p}^{T}+j \beta_{p} \mathbf{b}_{t, p}^{T}\right)=\mathbf{C}[t] \mathbf{s}
$$

where $\mathbf{s}=\left[\alpha_{1}, \ldots, \alpha_{P}, \beta_{1}, \ldots, \beta_{P}\right]^{T}, \quad \mathbf{C}[t]=$ $\left[\mathbf{a}_{t, 1}^{T}, \ldots, \mathbf{a}_{t, P}^{T}, j \mathbf{b}_{t, 1}^{T}, \ldots, j \mathbf{b}_{t, P}^{T}\right]$, and $\mathbf{a}_{t, p}$ and $\mathbf{b}_{t, p}$ are the $t$-th row of $\mathbf{A}_{p}$ and $\mathbf{B}_{p}$. Substituting (11) into (10), we get

$$
\mathbf{r}[t]=\mathbf{H}^{T}[t] \mathbf{C}[t] \mathbf{s}+\mathbf{w}[t], \quad t=1, \ldots, T .
$$

Stacking all $T \mathbf{r}[t]$ 's into a vector, we have

$$
\tilde{\mathbf{r}}=\tilde{\mathbf{H}} \mathbf{s}+\tilde{\mathbf{w}}
$$

$\begin{array}{ll}\text { where } \tilde{\mathbf{r}} & =\left[\mathbf{r}^{T}[1], \ldots, \mathbf{r}^{T}[T]\right]^{T}, \quad \tilde{\mathbf{H}}= \\ {\left[\mathbf{C}[1]^{T} \mathbf{H}[1], \ldots, \mathbf{C}[T]^{T} \mathbf{H}[T]\right]^{T}} & \text { and }\end{array}$ 
$\left[\mathbf{w}^{T}[1], \ldots, \mathbf{w}^{T}[T]\right]^{T}$. Assuming the additive noise is white Gaussian, the ML detector for STBC is given by

$$
\hat{\mathbf{s}}=\underset{\mathbf{s}}{\arg \min }\|\tilde{\mathbf{r}}-\tilde{\mathbf{H}} \mathbf{s}\|^{2} .
$$

The quadratic form (14) is similar to the quadratic ML cost function that occurs in a spatial-multiplexing system (e.g., BLAST) over a flat, static MIMO channel. Therefore, one may use suboptimal detectors such as the zero forcing (ZF) detector and the minimum mean square error (MMSE) detector that have previously been developed for the BLAST system to solve the quadratic form (14). For NOSTBC (8) with one receive antenna, we can show that the $\mathrm{ZF}$ detector for (14) reduces to the $\mathrm{ZF}$ detector in [6]. However, unlike the latter, the ZF detector for (14) can be applied to systems with an arbitrary number of receive antennas. New detector structures for (14) can also be developed using SD [8], which will be optimal, and the V-BLAST detector [12], which will be suboptimal but has better performance and higher complexity than the $\mathrm{ZF}$ and MMSE detectors. In the following, we give a brief review of the SD and V-BLAST algorithms.

SD takes advantage of the lattice structure of transmitted signals to achieve exact ML performance (i.e., the performance of exhaustive search) with moderate complexity. SD generalizes the nulling and cancelling, which forms the basis of the V-BLAST detector, where at each step, one considers all the signal vectors $\mathbf{s}$ that lie within a sphere of certain radius $r$, or equivalently SD solves

$$
\|\mathbf{M} \mathbf{s}\|^{2}<r^{2}
$$

Searching all of the candidates satisfying (15) forms a tree. When the search goes to the bottom of the tree or a valid candidate $\mathbf{s}_{c}$ is found within the sphere, $r^{2}$ is updated to $\left\|\mathbf{M} \mathbf{s}_{c}\right\|^{2}$ if the latter is smaller. With the properly chosen initial radius $r$, the complexity can be reduced significantly. Usually, the initial radius $r$ can be chosen according to the noise variance or the bound given by the V-BLAST solution. For complex square QAM constellations, $\alpha_{p}$ and $\beta_{p}$ form independent real pulse amplitude modulation (PAM) constellations. As a result, the complex SD problem will reduce to a larger real SD problem. However, for $M$-PSK $(M>4)$, this does not hold. $\alpha_{p}$ and $\beta_{p}$ are still constrained by the constellation. The details of a complex SD implementation are given in [11]. A similar idea can also be applied to the V-BLAST algorithm. Another consideration is that both SD and V-BLAST require the matrix $\tilde{\mathbf{H}}$ be of full column rank, which is equivalent to $T N_{r} \geq 2 P$. When $T N_{r}<2 P$, (13) becomes a rank-deficient system and both SD and V-BLAST cannot be directly applied. In [9], we have given an efficient solution to the rank deficient system, where both SD and V-BLAST can be applied.

We now consider the special case of OSTBC systems. In this case, the correlation between the $i$-th and $j$-th columns $(i \neq j)$ of $\tilde{\mathbf{H}}$ can be written as

$$
\phi_{i, j}=\tilde{\mathbf{h}}_{i}^{H} \tilde{\mathbf{h}}_{j}=\sum_{t=1}^{T} \mathbf{a}_{t, i}^{*} \mathbf{H}^{*}[t] \mathbf{H}^{T}[t] \mathbf{a}_{t, j}^{T} .
$$

When the channel is static during each codeword, (16) can be further simplified as

$$
\begin{aligned}
\phi_{i, j} & =\sum_{t=1}^{T} \mathbf{a}_{t, i}^{*} \mathbf{H}^{*} \mathbf{H}^{T} \mathbf{a}_{t, j}^{T}=\operatorname{tr}\left(\mathbf{A}_{i}^{*} \mathbf{H}^{*} \mathbf{H}^{T} \mathbf{A}_{j}^{T}\right) \\
& =\operatorname{tr}\left(\mathbf{A}_{j}^{T} \mathbf{A}_{i}^{*} \mathbf{H}^{*} \mathbf{H}^{T}\right)=\mathbf{0},
\end{aligned}
$$

where the last equality comes from the property of OSTBC (6). In (16) and (17), we have assumed that $i, j \leq P$. Similar results can be obtained for other $i, j$. Therefore, the equivalent channel matrix $\tilde{\mathbf{H}}$ is orthogonal when the channel is static. However, when the channel is time-selective, (17) does not hold and $\tilde{\mathbf{H}}$ is not orthogonal. Assuming that Jakes' model is used, we can compute $E\left\{\left|\phi_{i, j}\right|^{2}\right\}$ in closed-form as

$$
\begin{aligned}
E\left\{\left|\phi_{i, j}\right|^{2}\right\}= & \sigma_{h}^{4} \sum_{t_{1}=1}^{T} \sum_{t_{2}=1}^{T} J_{0}^{2}\left(2 \pi f_{d} T_{s}\left(t_{1}-t_{2}\right)\right) \\
& \times \operatorname{tr}\left(\mathbf{a}_{t_{1}, i}^{T} \mathbf{b}_{t_{1}, j} \mathbf{a}_{t_{2}, i}^{T} \mathbf{b}_{t_{2}, j}\right) .
\end{aligned}
$$

Using the trace property and (6), it can be readily verified that $E\left\{\left|\phi_{i, j}\right|^{2}\right\}=0$ when $f_{d}=0$. When $N_{r}=1$ and the Doppler spread is not very large, it has been shown in [5] by simulation that $E\left\{\left|\phi_{i, j}\right|^{2}\right\}, i \neq j$ is small. Using the closedform expression (18), we can show that this also holds true when $N_{r}>1$. Therefore, $\tilde{\mathbf{H}}$ is "almost" orthogonal for small $f_{d}$, which motivates the development of an efficient suboptimal detector. We multiply both sides of (13) by $\tilde{\mathbf{H}}^{H}$ and obtain

$$
\mathbf{y}=\tilde{\mathbf{H}}^{H} \tilde{\mathbf{r}}=\tilde{\mathbf{H}}^{H} \tilde{\mathbf{H}} \mathbf{s}+\tilde{\mathbf{H}}^{H} \tilde{\mathbf{w}}=\boldsymbol{\Phi} \mathbf{s}+\mathbf{v} .
$$

Ignoring the fact that the noise vector $\mathbf{v}$ is not white Gaussian, the suboptimal detector is thus given by

$$
\hat{\mathbf{s}}=\underset{\mathbf{s}}{\arg \min }\|\mathbf{y}-\mathbf{\Phi} \mathbf{s}\|^{2} .
$$

Taking the fact that $\phi_{i, j}, i \neq j$ is small into account, we can apply the principle of PIC to solve (20). The PIC detector results in the following two steps [5]:

- Initialization: Set iteration number $k=0$ and obtain the initial data detection using

$$
\hat{s}_{i}^{(0)}=\underset{s_{i} \in \mathcal{Q}}{\arg \min }\left|y_{i}-\phi_{i, i} s_{i}\right|^{2}
$$

where $\mathcal{Q}$ is the constellation for $s_{i}$ and $y_{i}$ is the $i$-th entry of $\mathbf{y}$.

- Iteration: $k=k+1$ and we compute

$$
\mathbf{y}^{(k)}=\mathbf{y}-\boldsymbol{\Phi}_{\mathrm{ND}} \hat{\mathbf{s}}^{(k-1)}
$$

where $\boldsymbol{\Phi}_{\mathrm{ND}}=\boldsymbol{\Phi}-\operatorname{diag}(\boldsymbol{\Phi})$ comprises the non-diagonal entries of $\boldsymbol{\Phi}$. The data are detected using

$$
\hat{s}_{i}^{(k)}=\underset{s_{i} \in \mathcal{Q}}{\arg \min }\left|y_{i}^{(k)}-\phi_{i, i} s_{i}\right|^{2}
$$

where $y_{i}^{(k)}$ is the $i$-th entry of $\mathbf{y}^{(k)}$.

Note that the initial data detection $(k=0)$ is also called conventional detector [4]-[6]. Compared with the detectors given in [4]-[6] using PIC, the detector (14) is ML and can be applied to any OSTBC system, or even to all of the linear 
dispersion codes. The detector does not need to be designed for specific codes as in [4]-[6]. Unlike the detectors in [4]-[6] that work for only a single receive antenna, our new formulation of suboptimal PIC detector (20) can be applied to a system with an arbitrary number of receive antennas.

\section{Simulation Results}

We consider the $\mathcal{G}_{4}$ code in [2] and the NOSTBC system (8). QPSK is used throughout the simulation. The number of transmit antennas is set to four $\left(N_{t}=4\right)$. The time selective channel is simulated by sampling a continuous fading process via Jakes' model. The SNR is defined as $N_{t} N_{r} \sigma_{h}^{2} / \sigma_{n}^{2}$. The ML detector (14) with SD is compared with a conventional detector (CD) and a PIC detector, where the iteration number is denoted by $I$.

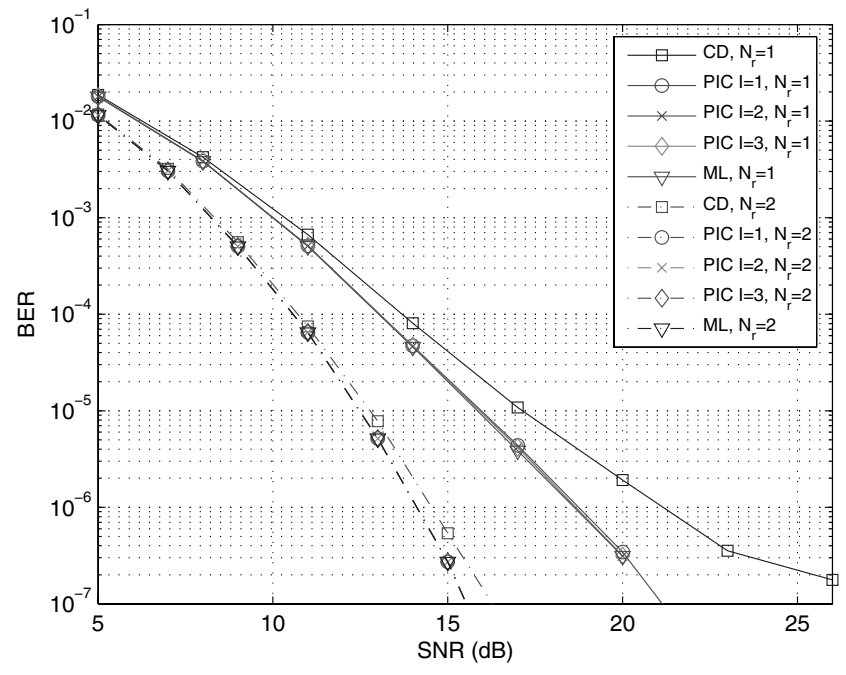

Fig. 1. BER versus SNR of different detectors for $\mathcal{G}_{4}$ code over a timeselective channel with $f_{d} T_{s}=0.005$.

Fig. 1 shows the BER performance of different detectors over a time-selective channel with $f_{d} T_{s}=0.005$. Both the performance of $N_{r}=1$ and $N_{r}=2$ is shown. When $N_{r}=1$, CD shows an error floor in high SNR. The PIC detector with $I \geq 1$ has almost the same performance as the ML detector in the observed region. Increasing $N_{r}$, the performance gap between $\mathrm{CD}$ and ML reduces. No error floor is observed. Therefore, introducing diversity can reduce the error floor.

When the Doppler spread increases to $f_{d} T_{s}=0.01$, both $\mathrm{CD}$ and PIC show error floors in high SNR as shown in Fig. 2. The error floor of PIC can be reduced by increasing the number of iterations $I$. However, performance gain diminishes by increasing $I$. The ML detector does not show an error floor, and it can exploit the full diversity of the system. Similarly, when increasing $N_{r}$, the performance of both PIC and CD improves, and the error floor is eliminated. The PIC detector with only one iteration achieves the performance of ML detector. The performance gap between $\mathrm{CD}$ and ML increases compared with the case $f_{d} T_{s}=0.005$.

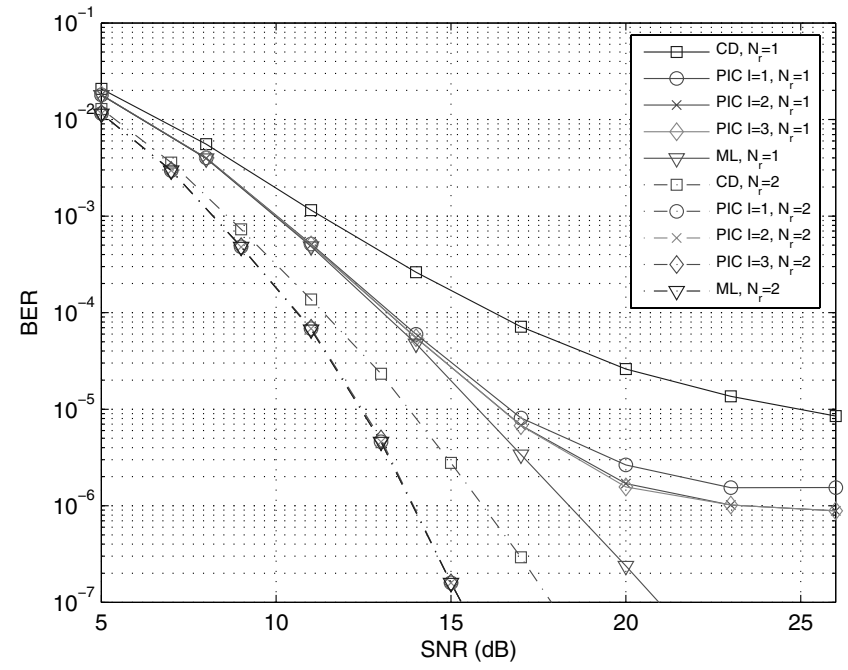

Fig. 2. BER versus SNR of different detectors for $\mathcal{G}_{4}$ code over a timeselective channel with $f_{d} T_{s}=0.01$.

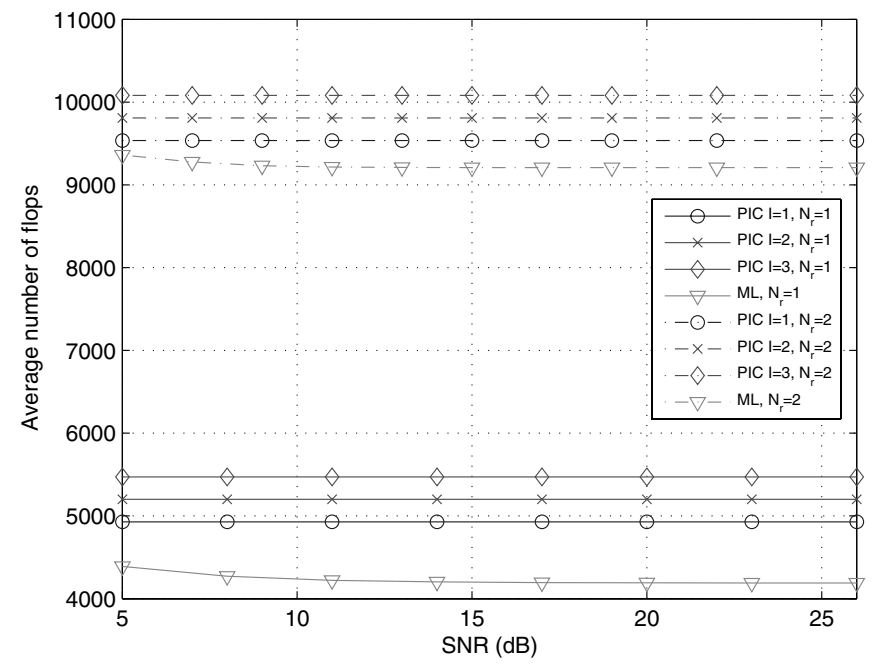

Fig. 3. Average number of flops versus SNR of different detectors for $\mathcal{G}_{4}$ code over a time-selective channel with $f_{d} T_{s}=0.01$.

Fig. 3 shows the average number of flops of different detectors over a time-selective channel with $f_{d} T_{s}=0.01$. The MATLAB V5.3 command "flops" is used to count the number of flops. Both the flops of the search stage and preprocessing stage in SD are counted. The complexity of PIC also includes the computation of $\mathbf{y}$ and $\boldsymbol{\Phi}$. For both $N_{r}=1$ and $N_{r}=2$, the complexity of PIC is higher than that of ML in both low and high SNRs. The complexity of both PIC and ML increases by increasing $N_{r}$ due to the increase of dimension in $\tilde{\mathbf{H}}$. The complexity of PIC increases by increasing $I$. The PIC detector can be easily parallelized as opposed to the SD. When a vector multiplication unit is available, the PIC is appropriate in low SNR and for a small Doppler spread. In high SNR, or for a large Doppler spread, the ML detector with SD is still a 
preferable choice since it eliminates the error floor.

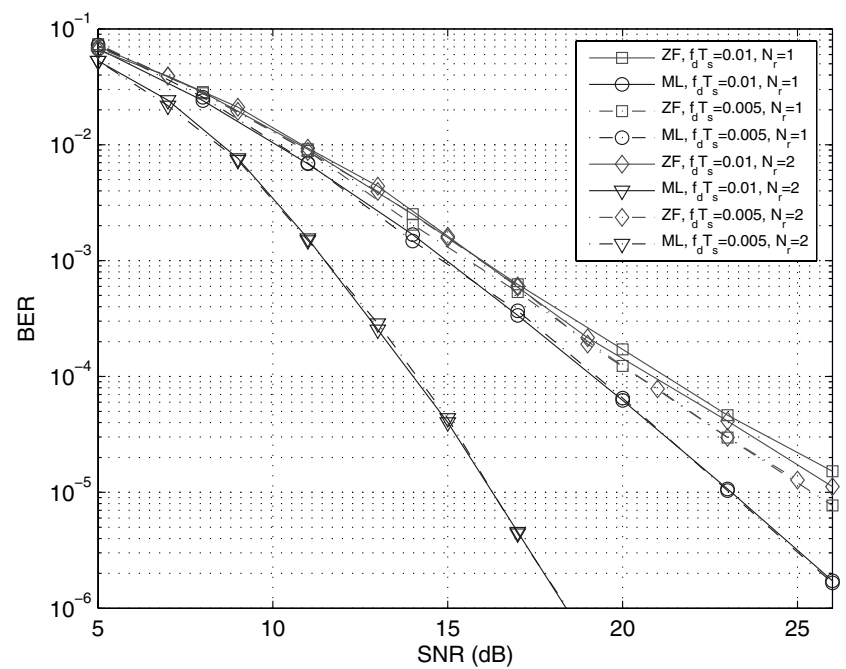

Fig. 4. BER versus SNR of different detectors for NOSTBC over a timeselective channel with different number of receive antennas and Doppler spreads.

Fig. 4 presents the performance of NOSTBC (8) of ML and $\mathrm{ZF}$ detectors over a time-selective channel with different $f_{d} T_{s}$ and different number of receive antennas. Note that when $N_{r}=1, T N_{r}<2 P$ and (13) is a rank-deficient system. We thus apply the generalized sphere decoder in [9] to solve (14). Increasing Doppler spread, the ML detector performs identically. The ZF detector with lower Doppler spread has better performance than that with higher Doppler spread. When $f_{d} T_{s}=0.005$ and $N_{r}=1$, the ML detector has a $2.5-\mathrm{dB}$ gain over the ZF detector. The diversity order of ML detector increases by increasing the number of receive antennas. However, the performance of $\mathrm{ZF}$ detector remains the same. Thus, the ZF detector cannot exploit the full diversity of the systems. When multiple receive antennas are employed in the system, our ML detector with SD is a preferable choice over time-selective channels.

\section{CONCLUSION}

We have investigated signal detection for STBC over timeselective fading channels. A general ML decision rule for STBC was derived, which resulted in a quadratic optimization problem that can be efficiently solved using the SD and the V-BLAST detectors. For orthogonal STBC systems with an arbitrary number of receive antennas, considering the almost orthogonal channel matrix, we also proposed a suboptimal detector using the principle of PIC.

\section{REFERENCES}

[1] S. M. Alamouti, "A simple transmit diversity technique for wireless communications," IEEE J. Select. Areas Commun., vol. 16, no. 8, pp. $1451-1458$, Oct. 1998.

[2] V. Tarokh, H. Jafarkhani, and A. Calderbank, "Space-time block codes from orthogonal designs," IEEE Trans. Inform. Theory, vol. 45, no. 5, pp. 1456 - 1467, July 1999.

[3] H. Jafarkhani, "A quasi-orthogonal space-time block code," IEEE Trans. Commun., vol. 49, no. 1, pp. 1 - 4, Jan. 2001.

[4] F.-C. Zheng and A. Burr, "Orthogonal space-time block coding over time-selective fading channels: a PIC detector for the H/sub i/ systems," in Proc. of ICC, Jun. 2004, pp. 687 - 691.

[5] - "Signal detection for orthogonal space-time block coding over time-selective fading channels: a PIC approach for the G/sub i/ systems," IEEE Trans. Commun., vol. 53, no. 6, pp. 969- 972, Jun. 2005.

[6] — "Signal detection for non-orthogonal space-time block coding over time-selective fading channels," IEEE Commun. Lett., vol. 8, no. 8, pp. 491 - 493, Aug. 2004.

[7] B. Hassibi and B. M. Hochwald, "High-rate codes that are linear in space and time," IEEE Trans. Inform. Theory, vol. 48, no. 7, pp. 1804 - 1824, July 2002.

[8] U. Fincke and M. Pohst, "Improved methods for calculating vectors of short length in a lattice, inlcuding a complexity analysis," Math. Computation, vol. 44, pp. 463-471, Apr. 1985.

[9] T. Cui and C. Tellambura, "An efficient generalized sphere decoder for rank-deficient MIMO systems," IEEE Commun. Lett., vol. 9, no. 5, pp. 423 - 425, May 2005.

[10] — "Approximate ML detection for MIMO systems using multistage sphere decoding," IEEE Signal Processing Lett., vol. 12, no. 3, pp. 222 - 225, Mar. 2005.

[11] - "Joint channel estimation and data detection for OFDM systems via sphere decoding," in Proc. of GLOBECOM, Dec. 2004.

[12] G. D. Golden, G. J. Foschini, R. A. Valenzuela, and P. W. Wolniansky, "Detection algorithm and initial laboratory results using the V-BLAST space-time communication architecture," Electron. Lett., vol. 35, no. 1, pp. 14-15, Jan. 1999.

[13] M. Enescu and V. Koivunen, "Time-varying channel tracking for spacetime block coding," in Proc. of VTC Spring, May 2002, pp. $294-297$. 